\title{
INFORMACIÓN BIBLIOGRÁFICA
}


ISSN: $1130-2887$

Ludolfo Paramio y Marisa Revilla. Una nueva agenda de reformas políticas en América Latina. Madrid: Fundación Carolina y Siglo XXI, 2006. 266 pp. ISBN 84-323-1256-8.

El libro editado por Ludolfo Paramio y Marisa Revilla Una nueva agenda de reformas políticas en América Latina analiza la situación a la que se enfrentan los países latinoamericanos después de una década -la de 1990- de profundas reformas estructurales. Es una compilación de las ponencias presentadas en el seminario que, bajo el mismo título, organizaron de forma conjunta la Fundación Carolina y la Universidad Internacional Menéndez Pelayo en agosto de 2005.

Contiene capítulos de diez autores que indagan en los resultados de las reformas implementadas por los países de la región durante la década pasada, las problemáticas a las que se enfrentan los actores políticos y sociales y los retos más importantes que les depara el futuro. Cada uno de estos trabajos se ocupa de un tema particular; desde las consecuencias que han tenido en el ámbito económico las reformas estructurales, pasando por los déficits en la construcción del Estado de Derecho, las crisis de gobernabilidad y la importancia del populismo para la política latinoamericana, el problema del desarrollo, la pobreza y las desigualdades sociales o las formas en las que la cooperación internacional contribuye a solventar algunas de estas dificultades.

En el primer capítulo, «América Latina después de las reformas», José Déniz Espinós analiza, desde una perspectiva crítica de las reformas y sus fundamentos, lo incompleto de la agenda y las debilidades en las que se asentó el programa de liberalización económica en la región, para concluir sobre la necesidad urgente de reformar las actuales reformas, en aras de mejorar el desempeño económico y de ubicarlas en un contexto de transformación más amplio que responda a un enfoque más integral y con perspectiva de medio y largo plazo.

Pilar Domingo, en su capítulo «Calidad de la democracia, ciudadanía y construcción del Estado de Derecho», ofrece interesantes reflexiones sobre los conceptos de ciudadanía y Estado de Derecho en el contexto del debate sobre la calidad de la democracia planteado por Guillermo O'Donnell en el Informe del Programa de Naciones Unidas para el Desarrollo (PNUD). La autora revisa los conceptos y observa la experiencia de construcción de ciudadanía en América Latina, desde la perspectiva de los derechos que la constituyen. Finalmente, plantea algunos de los problemas más importantes que supone para los países de la región el reto de fortalecer el Estado de Derecho. 
Con un enfoque que pone el acento en lo sustantivo, más que en lo procedimental, Ludolfo Paramio aborda en el capítulo «Crisis de gobernabilidad y populismo» el problema de la aparición de liderazgos populistas como consecuencia de la crisis de representación que sufren los partidos políticos latinoamericanos. Entendida como «una inadecuación funcional de los partidos -y como consecuencia, de los gobiernos que éstos forman- respecto a lo que los ciudadanos esperan o demandan», esta crisis representativa actual es vinculada por el autor a los cambios en las reglas de juego provocados por las reformas económicas de los años noventa, por una nueva relación de los partidos con los agentes de movilización social y por las limitaciones subjetivas de los propios partidos. Según Paramio, esta crisis de representación conforma el contexto más favorable para la aparición de líderes populistas, que a su vez contribuyen a profundizar el descrédito de las organizaciones partidistas existentes y al desmantelamiento o la perversión de las instituciones democráticas.

«Brechas de desarrollo y reforma institucional en América Latina», el capítulo de Joan Prats, comienza ofreciendo datos sobre la evolución del crecimiento económico, indicadores de desarrollo humano, de pobreza e indigencia, del peso de la economía informal y de la desigualdad social. Posteriormente, recorre el nacimiento de los Estados latinoamericanos hasta llegar a la última ola de democratización vivida por la región, que corrió paralela a la implementación de las políticas del Consenso de Washington -a las que dedica especial atención-, lo que le permite afirmar que la falta de desarrollo institucional y la inseguridad jurídica son los mayores riesgos que amenazan a las sociedades actuales y a sus procesos de inserción en la economía internacional.

Fernando Carrillo-Flórez analiza la importancia de recuperar la centralidad de la política como forma de revertir las deficiencias experimentadas por los países latinoamericanos y de superar la insatisfacción con la democracia, a la que se ha convertido en chivo expiatorio de décadas de inequidad en la región. Examina una combinación de múltiples dimensiones que han sido malas compañeras de viaje en América Latina y que se han traducido en problemáticas para el desarrollo económico, político y social y propone, como una de las posibles formas de hacer frente a estos dilemas, la reconciliación entre la política y la economía en el marco del fortalecimiento de las instituciones del Estado democrático de Derecho y la participación de la sociedad civil. Considera que una mayor institucionalización de la vida pública que contribuya a hacer frente a los desequilibrios sociales y a las transformaciones económicas es, en este sentido, la forma de potenciar la democracia.

El único capítulo que analiza un caso particular es el de Teresa Ossio Bustillos. El capítulo «Movimientos sociales y nuevas protestas: Bolivia» analiza los escenarios de movilización y crecimiento social en la historia boliviana hasta llegar al último, en el año 2000. Para comprender el funcionamiento de los nuevos movimientos sociales y de las situaciones de ingobernabilidad a las que ha estado sometido el país desde principios del siglo XXI, Ossio repasa su historia poniendo énfasis en diferentes crisis y sucesivos movimientos generacionales que detentaban sus propios proyectos de poder y de país. Por último, se detiene en las características y singularidades que muestran en la actualidad las formas de acción colectiva y el contexto en el que se expresan. 
«Las élites frente a la crisis del sistema político» de Manuel Alcántara Sáez presta especial atención a la agenda de reforma política para la región tomando como punto de partida las opiniones de las élites parlamentarias latinoamericanas. Este trabajo indaga en las percepciones, actitudes y opiniones de los diputados con relación a los puntos más sensibles que constituyen los principales problemas a los que se enfrenta la política en cada uno de los países. Alcántara concluye su trabajo señalando que los aspectos centrales que los políticos apuntan como principales son los de carácter social y económico en mayor medida que los que tienen que ver con la estricta reforma política.

El estudio comparado de las reformas políticas-electorales en la región es el tema del capítulo «La reforma político electoral en América Latina. Evolución, situación actual y tendencias, 1978-2005» de Daniel Zovatto. Este análisis cubre los 18 países de la región y está organizado en torno a tres ejes principales: a) las reformas del régimen de gobierno; b) las reformas del sistema electoral; y c) las reformas de los partidos políticos, en materia de financiación interna y financiación.

«América Latina entre la angustia y la esperanza» de Salvador Samayoa aborda tres cuestiones trascendentes en la agenda latinoamericana: la seguridad, la violencia y el desarrollo en la región. El capítulo comienza con un panorama general de la situación que viven los ciudadanos, caracterizada por la violencia y la delincuencia; continúa con un examen de las políticas de integración social que sería necesario impulsar y finaliza con las reformas institucionales más importantes que deberían emprenderse o fortalecerse para superar estas problemáticas (principalmente las reformas que se refieren al tema de la seguridad pública y la justicia, la descentralización y el fortalecimiento municipal, y la educación).

El último capítulo de este libro es de Aurora Díaz-Rato y se titula «La cooperación española y la gobernabilidad democrática en América Latina». En él se destaca la apuesta de la cooperación española para apoyar el fortalecimiento de las instituciones democráticas en la región y el nuevo paradigma de sus programas en el que convergen la consolidación democrática y el desarrollo sobre la base de que las instituciones son cruciales para el desarrollo sostenible con equidad y que el Estado debe ser reconstituido para poder desempeñar sus responsabilidades. El trabajo de Díaz-Rato enuncia y analiza los principios de actuación de la cooperación española en materia de gobernabilidad democrática y las áreas sectoriales de intervención, al mismo tiempo que plantea otras áreas transversales priorizadas como son la integración regional, la profesionalización de la función pública, la inclusión política, la generación de consensos políticos, la promoción de las TICS y el apoyo a la creación de redes como instrumento de política y comunicación, por nombrar sólo algunas.

Todos los autores que participan de este libro coinciden en reforzar la idea de una imperiosa necesidad de fortalecer las instituciones públicas para acometer el desafío de mejorar la calidad de la política y de la democracia. Al mismo tiempo, ya sea basándose en las consecuencias de la experiencia reformista de la década de 1990 o en los problemas que en la actualidad son más visibles, afirman que las decisiones sobre las transformaciones que deben tener lugar en los países deben sustentarse en 
las particularidades de cada uno de ellos; es decir, teniendo en cuenta el contexto en el que se implementan.

La revitalización de la política como variable fundamental a considerar para hacer frente a «la reforma de las reformas» y la insistencia en la dimensión institucional, en tanto reglas de juego que condicionan las transformaciones y los nuevos desafíos, son el común denominador de estos trabajos de reconocidos expertos del mundo académico, de los organismos internacionales y de la cooperación. Una nueva agenda de reformas políticas en América Latina es, en este sentido, un libro necesario para comprender los grandes problemas pendientes en el plano social e institucional y para pensar y reflexionar hacia dónde deberían ir encaminadas las nuevas reformas en la región.

Patricia MARENGHI

Dieter Nohlen. Diccionario de Ciencia Política. Ciudad de México: Editorial Porrúa México y El Colegio de Veracruz. 2 volúmenes, 2005.785 y 1.529 pp. ISBN 97007-6115-0 (con la colaboración de Rainer-Olaf Schultze).

Con el entusiasmo de quien escribe su primer libro, el profesor Dieter Nohlen ha conseguido, luego de un arduo trabajo, que uno de sus mayores anhelos se hiciera realidad: la publicación de una versión en español de su reconocido Lexikon der Politikwissenschaft, que compiló con la colaboración del profesor Rainer-Olaf Schultze y que hasta el momento sólo se había publicado en alemán (2002). Esta versión del Diccionario de Ciencia Política no es una simple traducción de los vocablos publicados en el original sino que cuenta con nuevos conceptos, la sustitución y eliminación de otros, la adaptación de muchos de los términos a la realidad hispanoamericana así como también la adecuación de las referencias bibliográficas a los materiales que están accesibles en español.

El Diccionario es una obra que aborda las herramientas conceptuales, metodológicas y teóricas de la Ciencia Política de una manera sistemática. El objetivo es revisar el modo en que se han definido los conceptos, las metodologías que se han empleado y las teorías que se han utilizado para explicar diversos fenómenos políticos. Esto diferencia a la obra de otras enciclopedias de la política o incluso de otros Diccionarios de Política como el publicado por Norberto Bobbio, Nicola Mateucchi y Gianfranco Pasquino (Madrid: Siglo XXI, 1991). Aquí no se trata de estudiar fenómenos sino de revisar el modo en que se han estudiado e identificado las relaciones entre los diversos conceptos y los enfoques teóricos que le han dado sustento. Por tanto, este trabajo no es sólo un Diccionario de Política sino un manual, donde conceptos ordenados alfabéticamente se interrelacionan y referencian mutuamente.

El volumen I de la obra está precedido por un ensayo introductorio sobre cómo enseñar y cómo estudiar Ciencia Política. En el texto, Nohlen manifiesta su preocupación 
por el desarrollo de la Ciencia Política como disciplina científica; su vinculación con la evolución política de cada país y su papel como una especialidad estrechamente relacionada con la democracia, al punto de que resulta imposible su ejercicio sin un contexto de pluralismo y competencia política. Además, presenta un rico esquema respecto a la enseñanza de la disciplina, definiendo su campo de estudio, su método y su diferenciación de la política cotidiana. Este análisis resulta muy útil para ayudar a delimitar el ámbito de estudio de la Ciencia Política, en un contexto como el latinoamericano, donde durante décadas ha estado abandonada, debido a las interrupciones autoritarias y sultanísticas. Esto ha provocado que bajo su nombre se enseñaran contenidos que estarían lejos de su ámbito de interés, si se lo compara con el de universidades europeas o norteamericanas y ha fomentado un desarrollo desigual de la disciplina en la región, como bien se ha podido comprobar en la reciente investigación desarrollada y publicada por la Revista de Ciencia Política de Chile (2005).

En cuanto a la primera cuestión, el objeto de análisis de la Ciencia Política, se pueden diferenciar tres áreas. Primero, la de la teoría política, en la que a su vez se encuentran tres subáreas: la de la historia de las ideas, la de la teoría política moderna y la de la metodología en ciencias sociales. Segundo, la de la política comparada, que está subdividida en tres dimensiones: la de la forma, el proceso y el contenido de la política (lo que en inglés se diferencia como polity, politics y policy). Tercero, la de las relaciones internacionales, donde se incluye tanto la política exterior, las relaciones intergubernamentales y entre diversas manifestaciones de la sociedad civil o el nivel supranacional. Estas tres áreas no pueden analizarse de manera autónoma, sino que viven constantemente interrelacionadas. Por lo que aun cuando uno se especialice en una materia, el estudio siempre debe integrar a las demás.

En relación a la segunda dimensión, en cuanto a cómo enseñar Ciencia Política, el autor señala que se debe emplear el pensamiento lógico y sistemático, esto es, difundir la lógica de la argumentación, que permita diferenciar al interior de lo conceptual. Se plantea la necesidad de emplear el método comparado para la investigación en Ciencia Política. Urge implementar contenidos donde se enseñe la política comparada como algo clave en la investigación politológica. Nohlen alerta respecto a que en América Latina se ha desarrollado más la comparación histórico-cronológica y mucho menos la sistemático-comparativa. Por eso, resulta clave introducir asignaturas que enseñen a comparar a los estudiantes y que se desarrollen investigaciones a partir de emplear comparaciones internacionales sobre la base de tipologías y evidencias empíricas.

Finalmente, en el artículo introductorio, el autor destaca el hecho de que la reflexión científica se debe separar de la política cotidiana, del mundo de la opinión y del compromiso ideológico. La investigación politológica debe estar exenta de las pasiones partidistas o interesadas. La enseñanza de la disciplina debe estar en un nivel de abstracción lo más alto posible, como «algo lejano y fuera de la política» (p. xxiv), para de esa manera convertir a la política en objeto de estudio. Todo esto supone sustituir la «cultura de la opinión por la cultura del argumento» (p. xxv), consejo no desdeñable para algunos centros que dicen enseñar Ciencia Política en América Latina. 
Esta obra se convertirá en un libro de consulta obligada para profesores y estudiantes de Ciencia Política, por varias razones. En primer lugar, debido a la exquisita sistematización y rigurosidad con la que se ha trabajado cada uno de los conceptos. En segundo lugar, porque dado el conocimiento sobre América Latina del compilador, la obra ayuda a ubicar estos conceptos en la tradición teórica y empírica latinoamericana. En tercer lugar, porque tras la traducción al español, es posible ubicar el desarrollo bibliográfico de esos términos en materiales accesibles para el investigador hispano. Con ello, la obra permite tener a la mano un estado de la cuestión de las principales publicaciones realizadas sobre América Latina en diversas líneas de la investigación. Finalmente, el libro resulta un aporte excelente para el conocimiento de las herramientas metodológicas aplicables a la investigación politológica, dando cuenta del interés del profesor Nohlen por el desarrollo de una Ciencia Política lógicamente argumentada, metodológicamente rigurosa y sistemáticamente estructurada en la región.

Flavia FREIDENBERG

David SHIRK. Mexico's New Politics. The PAN and Democratic Change. London: Boulder, 2005. 279 pp. ISBN 1-528826-270-7.

La aparición de este trabajo responde a la imperiosa exigencia de entender cuáles fueron las condiciones que hicieron posible que el Partido Acción Nacional (PAN), que había fungido desde 1939 como el principal opositor dentro del sistema de partidos en México, pudiera finalmente derrotar después de un prolongado proceso de participaciones electorales en condiciones no competitivas a la hegemonía de la clase política encabezada por el Partido Revolucionario Institucional (PRI). Y, al mismo tiempo, trata de ofrecer claves de lectura acerca de los elementos que permitan entender si las capacidades y resultados del gobierno del presidente Vicente Fox se deben o no al apoyo y aplicación de los principios y programas promovidos por dicha instancia partidaria.

El PAN es visto por el autor desde la perspectiva de la construcción organizativa de coaliciones internas de grupos competitivos que poseen fines comunes, en este caso, superar las condiciones no democráticas prevalecientes dentro del sistema político. El autor dedica particular atención al punto de entender la conformación de la matriz organizativa del partido, en tanto reconoce a las fuentes doctrinarias de corte liberal-conservador y católico-humanista, como los dos principales pivotes que articularon a las diversas luchas ciudadanas de la militancia, sobre todo en la defensa y reconocimiento de sus votos.

Ambas corrientes no han estado exentas de conflictos e incluso han sido testigos de rupturas y escisiones que colocaron por momentos al partido en el borde de un cisma final, sobre todo en la década de los setenta, en tanto la diferencia se colocó en optar por el pragmatismo y colaboración para ir obteniendo posiciones, o ir abiertamente a 
una estrategia antisistémica que propiciara la caída total del régimen. Sin embargo, resulta interesante observar que la fuerza de los incentivos internos y externos permitió al PAN mantenerse en la línea central de su objetivo histórico de llegar a obtener la presidencia mexicana por la vía electoral y civil.

Sin embargo, dichos avances no fueron simplemente acumulativos. Por otra parte, el autor hace una puntual revisión de la coyuntura que propició dicho incremento y desafección al sistema, caracterizada por una creciente ineficacia económica y el desgaste histórico experimentado por el PRI hacia mediados de los años ochenta, los cuales pueden ser considerados como el principal detonador de la crisis que impulsa al éxito electoral del PAN en diversas zonas del país, lo cual terminó por dar la razón a quienes decidieron «abrir» y moderar al partido con la incorporación de nuevos militantes y recursos, aunque éstos en un primer momento no tuvieran el perfil sociocultural ni la formación ideológica usualmente requeridas por el propio PAN.

Por otra parte, el autor define que el desarrollo del PAN ha sido uno con características que le hizo moverse desde las periferias y que alcanzaron la cúspide en el año 2000 aunque sigue sin llegar a transformar y dominar por entero a toda la base y las estructuras intermedias. De alguna forma, la presencia de un partido que debe adaptarse y ubicarse conforme va avanzando el nuevo contexto de cambio y negociación entre las fuerzas políticas, remite además también a las exigencias de contar con reglas e instituciones eficaces, las cuales deben ser ahora impulsadas y reformadas precisamente mediante acciones democratizadoras y descentralizadoras del poder público.

En este aspecto, el autor también procura abordar los retos que han enfrentado las diversas áreas de política pública durante la gestión de Vicente Fox. Si bien se pudo iniciar con importantes cuotas de capital social de apoyo, las bases de movilización y cooperación institucional se volvieron insuficientes, en tanto el PAN no obtuvo la mayoría parlamentaria en las cámaras, ni tampoco pudo ser exitoso en la conformación de un gabinete de colaboradores que pudiera avanzar en la aplicación de los programas propuestos, cuestión que se fue acentuando después del fracaso electoral de las elecciones intermedias del 2003, las cuales todavía acotaron más la acción del presidente y acrecentaron su distanciamiento efectivo respecto del propio PAN.

Es evidente que la posibilidad de generar modificaciones radicales en sólo seis años resulta ilusoria. Sin embargo, el autor es acertado al considerar que la mayor aportación generada por el PAN ha sido la de empujar una ruta de introducción a las prácticas institucionales democráticas, aunque dicho marco de aprendizaje y éxito necesariamente implica la participación y el compromiso público de los demás actores políticos y sociales para poder emprender acciones de largo plazo. De ahí que la posibilidad de continuidad democrática en México no pueda depender de un solo partido o individuo, por más providenciales que éstos sean, sino de la presencia real de instituciones sólidas y responsables.

Víctor AlarCón Olguín 
Kristen SAmple y Daniel ZovatTo (eds.). Democracia en la Región Andina: los telones de fondo. Estocolmo: International Institute for Democracy and Electoral Assistence, 2005. 300 pp. ISBN 91-85391-53-0.

Democracia en la Región Andina: los telones de fondo es el segundo de una serie de trabajos orientados al estudio de la situación de los Países Andinos, después de más de 25 años de democratización. Surge de una iniciativa conjunta del Instituto Internacional para la Democracia y la Asistencia Electoral (IDEA) y de la Asociación Civil Transparencia. El objetivo de este trabajo es elaborar una panorámica de la situación de las democracias andinas. Los trabajos presentados desde diversos enfoques teóricos y metodológicos plantean la cuestión de cómo reorganizar la vida política en contextos altamente conflictivos y débilmente institucionalizados.

El primer trabajo, «Valores, percepciones y actitudes hacia la democracia. Una visión comparada en la Región Andina: 1996-2004», realizado por Daniel Zovatto, analiza las percepciones sobre la democracia que tienen los ciudadanos y los bajos índices de satisfacción con su funcionamiento y de confianza en las instituciones. La principal conclusión a la que se llega es que el desafío más importante de los países andinos es la profundización de la ciudadanía y la consolidación de una cultura política democrática. En «Los sistemas de partidos en los países andinos: autoritarismos competitivos y reformismo institucional», Martin Tanaka muestra cómo en países como Venezuela y Perú las pugnas internas en los partidos tradicionales quebraron las estructuras de los sistemas partidistas dando paso a la instauración de autoritarismos competitivos; mientras que en Ecuador, Bolivia y Colombia los sistemas lograron evolucionar en medio de la crisis gracias a que los partidos mantuvieron sus estructuras y optaron por estrategias de reformismo institucional orientado al reconocimiento de nuevos derechos y actores.

En «Selección de candidatos, partidos y democracias en la Región Andina», Flavia Freidenberg resalta la importancia del funcionamiento interno de los partidos políticos en la mejora del rendimiento de los sistemas democráticos, centrándose en los procesos de selección de candidatos en veinte partidos de la región. Los resultados arrojados por el estudio dejan de manifiesto la relevancia y recurrencia de las relaciones y procedimientos informales al interior de los partidos, que limitan la participación de un mayor número de actores, el distanciamiento del partido por los intereses sociales, la resolución de conflictos entre múltiples liderazgos y la posibilidad de aumentar el nivel de legitimación del partido. El trabajo titulado «El financiamiento de los partidos políticos y las campañas electorales en los países andinos: Bolivia, Colombia, Ecuador, Perú y Venezuela», escrito por Humberto Njaim, presenta una panorámica comparada del funcionamiento real de los Sistemas de Financiamiento Político (SFP); frente a la existencia de numerosos elementos que obstaculizan su institucionalización, se resalta el control social que ejercen nuevos actores sociales y los avances en el funcionamiento interno más gerencial de los partidos. 
En «La representación política de las mujeres en la Región Andina», Kristen Simple analiza la participación de las mujeres en el poder legislativo en los países de la región en el marco de la discusión sobre la crisis de representatividad en cuyo centro se encuentran los partidos políticos. En este mismo sentido, en «Participación política y empoderamiento indígena en la Región Andina», Wigberto Rivero Pinto hace una reconstrucción histórica de la forma en que los indígenas han incursionado en el ámbito político. En la complejidad de este contexto, el autor plantea la posibilidad de construir democracias multiétnicas, en donde el poder ya no «exprese únicamente los intereses de las élites dominantes».

Desde una perspectiva institucional, en «Sistemas electorales en la Región Andina», Fernando Tuesta Soldevilla se aproxima al problema de la gobernabilidad en los Países Andinos a través del análisis de las reformas electorales, mostrando sus posibilidades en la generación de vías de canalización de conflictos sociales. En «La participación electoral en la Región Andina, 1978-2004», Daniel Zovatto estudia los factores que inciden en la participación electoral, establece la relación entre dicha participación, el índice de libertades democráticas y la percepción del proceso electoral, y analiza la participación electoral de cada país.

Entre tanto, en «Democracia directa en los países de la Región Andina. Efectos en la consolidación de la democracia», Humberto De La Calle analiza el funcionamiento y los alcances que ha tenido el uso de mecanismos de participación directa. Como en los anteriores estudios, sobresale el desencanto de la población con el funcionamiento del sistema democrático, el reforzamiento en algunos casos de tendencias populistas y la inmadurez de las instituciones. Por su parte, en el trabajo «Pactos interpartidarios y diálogos político-sociales en la Región Andina» de Rafael Roncagliolo y Ramón Ponce Testino, estudian las experiencias de concertación política, centrándose en los pactos en Bolivia, Colombia y Venezuela y en los diálogos político-sociales en Bolivia, Perú, Ecuador y Venezuela. Los autores resaltan el carácter consensual y deliberativo de la democracia, concluyendo que los pactos partidistas aunque supusieron una mayor estabilidad del sistema, generaron un alto índice de exclusión, que posibilitó la emergencia de nuevas fuerzas con discursos antisistémicos.

En cuanto al papel de la sociedad civil, en «La participación política de la sociedad civil en la Región Andina», Luis Verdesoto y Gloria Ardaya resaltan su importancia en la consolidación democrática, planteando, a su vez, la necesidad de reflexionar sobre nuevos retos para el fortalecimiento de vías institucionales a través de las cuales se canalice el descontento social. Finalmente, en «Agendas distantes: los medios de comunicación y los partidos políticos en la Región Andina», Santiago Pedraglio muestra la forma en que en las últimas décadas los medios de comunicación han pasado a ser intermediarios entre la ciudadanía y los partidos políticos. Lo que está en el centro del debate es el problema de la representación y la necesidad de una nueva legitimidad estatal.

En resumen, el texto comentado es una valiosa contribución a la comprensión de la realidad andina, ya que, al mismo tiempo que muestra un rigor conceptual y metodológico en cada artículo, explora los principales problemas que aquejan a la región y las posibilidades de profundizar en su democratización, aportando a su vez una rica 
base empírica, lo que aumenta su valía. Este trabajo está dirigido a todo tipo de público ya que el planteamiento de los problemas así como su desarrollo se elabora de manera clara y concisa permitiendo crear una panorámica política general de la región.

Lina María CABEZAS RinCÓN

Salvador MARTí I PUIG y Carlos Figueroa IbARRA (eds.). La izquierda revolucionaria en Centroamérica. De la lucha armada a la participación electoral. Madrid: Editorial Catarata, 2006. 223 pp. ISBN 84-8319-251-9.

Este trabajo presenta un completo estudio sobre los cambios acontecidos en las fuerzas de izquierda centroamericanas desde sus inicios, como organizaciones armadas, hasta la actualidad, como partidos políticos imbuidos en otro tipo de lucha. El libro se centra en las tres fuerzas más representativas: el Frente Sandinista de Liberación Nacional (FSLN) en Nicaragua; el Frente Farabundo Martí para la Liberación Nacional (FMLN) en El Salvador y la Unidad Revolucionaria Nacional Guatemalteca (URNG). Las mutaciones y cambios producidos son analizados desde el punto de vista organizativo, programático y discursivo. El libro se sostiene en importantes trabajos de investigación realizados por los autores y que aquí encuentran un sentido unitario que los potencia.

En el primer capítulo, Salvador Martí i Puig presenta un estudio de características generales que se convierte en una excelente arqueología, en clave comparada, de los movimientos guerrilleros, a través de la cual desentrañar las causas del diferente devenir de cada una de estas organizaciones. Aporta, además, una mirada sobre el presente y el futuro, exponiendo los problemas que se vislumbran en la izquierda revolucionaria fruto de su inserción como fuerza político-electoral.

En los siguientes tres capítulos se procede al análisis de cada uno de los casos mencionados. En el capítulo dos, Salvador Martí i Puig y Salvador Santiuste Cué hacen balance de la trayectoria del FSLN desde sus orígenes hasta tiempos presentes. Aquí se observan dos cuestiones básicas. La primera es que los cambios más profundos se producen en su estructura, profundamente afectada por las sucesivas transformaciones que la llevan de ser una organización política-militar, a partido-Estado y, finalmente, partido volcado a la competencia electoral. La segunda cuestión es que a pesar de los cambios mencionados no se han producido reemplazos en el liderazgo encarnado en el ex presidente Daniel Ortega.

En el capítulo siguiente, Alberto Álvarez realiza una panorámica del itinerario político del FMLN. Tratando sus antecedentes, su acción, la conversión en la fuerza guerrillera más numerosa de América Latina, su evolución durante y luego de los Acuerdos de Paz, finalizando en su accionar como fuerza política en democracia y los conflictos internos que agudizan el férreo control interno de la Comisión Política 
y la imposibilidad de llegar al poder en el corto plazo. En el tercer capítulo, Carlos Figueroa Ibarra aborda la trayectoria de la URNG mostrándola de una manera bastante diferente a los antes analizados. Se presenta la hipótesis que el fin de la guerra y la caída de la utopía revolucionaria condujeron a la crisis actual que vive la izquierda guatemalteca. El desafío de superar el estancamiento parece venir de la mano de encarar procesos de renovación sin perder las señas de identidad.

En el quinto capítulo, Salvador Martí i Puig y Salvador Santiuste Cué comparan las percepciones de los dirigentes de las tres fuerzas políticas y de los de partidos de derecha presentes en los mismos países. Se trata de observar qué diferencias persisten en la actualidad entre grupos que en el pasado se encontraban en las antípodas. Resulta significativo que ambos grupos coincidan en que la democracia representativa es el mejor de los regímenes y que sus reglas de juego son aceptables. Sin embargo, estas democracias tan duramente conseguidas han desembocado en un proceso que sólo favorece a unas elites políticas que no dan respuestas a las demandas que, tres décadas atrás, pusieron en crisis a los regímenes autoritarios. A modo de cierre, en las conclusiones, Salvador Martí i Puig y Carlos Figueroa Ibarra repasan los antecedentes y las trayectorias de los actores analizados y presentan algunos de los desafíos para las formaciones de izquierda aquí analizadas.

Los autores afirman que, más allá de las contradicciones y los cambios, esta izquierda aún mantiene diferencias sustantivas con la derecha y que en el futuro deberá elaborar un ideario de manera más precisa, no sólo como un recurso de identidad. Entre los méritos de este libro, algunos mencionados, retomamos lo expuesto en la presentación realizada por Manuel Alcántara. Este libro rescata un espacio y un objeto de estudio. Rescata a Centroamérica del doble olvido en que cayeron los estudios sobre la región, luego de la efervescencia inicial de los Acuerdos de Paz y, frente al nuevo auge de estudio de las izquierdas sudamericanas, que abarca sólo a aquellos partidos que han alcanzado el éxito electoral, esta obra toma en cuenta a actores que dieron pasos claves para la pacificación y democratización de sus países.

Fernando PEDROSA RAISKY

Guillermo EsCOBAR y Manuel GuEdán (eds.). Democracia e instituciones en América Latina. Madrid: Dykinson, 2005. 289 pp. ISBN 84-9772-658-8.

La democracia formal o procedimental presupone una mayor participación de los ciudadanos en los asuntos públicos que les conciernen. Éste es el punto de partida del presente libro: la consolidación de la democracia en América Latina a través de la potenciación de los mecanismos que implican a la ciudadanía en los temas públicos, reforzando en este sentido el papel catalizador que desempeñan las instituciones. Tomando como eje central esta definición restrictiva de democracia-democracia 
formal- los autores dejan a un lado otras acepciones del concepto democrático, como pudieran ser la justicia social $\mathrm{u}$ otros temas interesantes como el papel que ejercen los medios de comunicación en los sistema democráticos presentes, para centrarse en la democracia entendida como una serie de normas y procedimientos que aseguran la participación de la sociedad en la esfera pública. Resulta necesario advertir que el creciente descontento hacia la democracia en algunos países latinoamericanos, aunque también europeos, hace necesaria una reflexión sobre las causas del mismo. Propuestas que no tienen por qué conducir necesariamente a una mayor expansión y fortalecimiento del poder de los estados actuales, sino a una mejor configuración y adecuación de éstos a la sociedad, de modo que la ciudadanía encuentre adecuados canales públicos que les garanticen su participación.

El texto se estructura en once capítulos que se corresponden con los países latinoamericanos analizados -Argentina, Bolivia, Chile, Colombia, Costa Rica, Ecuador, El Salvador, Honduras, México, Paraguay y Perú- más un apartado final de conclusiones. A su vez, cada capítulo está dividido en cinco áreas temáticas que tienen como referencia el concepto de democracia formal antes mencionado. El primero de estos temas está referido a la libertad de asociación traducida en la libre pertenencia a los partidos políticos, analizándose de ellos su desarrollo orgánico, financiación, relación con otros poderes públicos, democracia interna y el derecho a asociarse. A continuación, se estudian los elementos de democracia directa como son la iniciativa y la consulta popular, el referéndum o el plebiscito, aportando mecanismos singulares como el derecho a la insurrección en El Salvador. De forma paralela, el tercer tema se centra en las vías de participación popular en ámbitos públicos específicos tales como el medio ambiente, la educación, la justicia, los medios de comunicación y los procedimientos administrativos. En cuarto lugar, se describen los sistemas electorales de cada país, los procedimientos electorales, los órganos de control de estos últimos, las formas de selección de candidatos y las garantías contra las irregularidades electorales. Las instituciones de la democracia representativa constituyen el último apartado. No sólo se estudian las instituciones clásicas de gobierno sino también las relaciones entre representantes y representados y la participación de los ciudadanos en la actividad legislativa. Al final de cada capítulo se agrega un breve epígrafe de consideraciones finales que trata de resaltar las principales deficiencias en materia de participación ciudadana en cada país, proponiendo algunas alternativas a las mismas.

El estilo que adopta el libro, excepto al final de cada capítulo y en el apartado de conclusiones, es esencialmente jurídico por lo que el contenido resulta muy descriptivo, pero tanto la sencillez del planteamiento del que se parte como su claridad conceptual hacen que su lectura pueda resultar de gran utilidad. Y esto tanto para quienes estén interesados en el estudio de las instituciones básicas de la democracia en Latinoamérica, como para quienes desean ir un poco más allá y quieren reflexionar en torno a la profundización de los regímenes democráticos en estos países.

En resumen, la idea que subyace en todo el texto es que si bien los pilares básicos de la democracia están asentados en América Latina, es necesaria la formulación de propuestas para avanzar en su afianzamiento. De entre las muchas alternativas para 
mejorar lo anterior, una de ellas pasa de forma apremiante por fomentar la implicación de la ciudadanía en los temas públicos. Es decir, los autores plantean implícitamente la necesidad de evitar el peligro de convertir los sistemas democráticos latinoamericanos en meras democracias de ciudadanos pasivos. La mejora de los mecanismos de participación existentes junto con la creación de otros, sería la plasmación real de esta idea que atribuye a las instituciones un papel relevante para conseguir el aumento de los medios de participación social. Todo ello sin sobredimensionar las facultades e instituciones del Estado, sino tratando de optimizarlas hacia la intervención activa de la sociedad.

David Molina Romo

Víctor EsPinOza VAlLE y Luis Rionda RAmíRez (eds.). Después de la alternancia: elecciones y nueva competitividad. México: Ediciones Eón S.A., 2005. 381 pp. ISBN 968-5353-64-6.

A pesar de que durante las cinco últimas décadas la transición política a la democracia en México ha ido acompañada de estudios realizados por el espacio académico, no ha sido hasta la década de 1990 que la academia ha comenzado realmente a preocuparse por los procesos político-electorales. Anteriormente, el entorno político se caracterizaba por la ausencia de competitividad y por la concentración de las capacidades reales de decisión sobre los temas públicos importantes en las manos de una limitada elite gobernante. Los momentos electorales no planteaban incógnitas y la renovación de los espacios de representación social ocurría mediante negociaciones que se llevaban a cabo por la elite perteneciente a los ejecutivos federal y estatal. En las elecciones, el voto popular era un simple acto simbólico por el que se legitimaban los nombramientos que previamente se habían acordado. Fue a partir de la emergencia de procesos electorales competitivos en ámbitos municipales y locales en la década de 1980, la primera alternancia estatal en 1989 en Baja California, las sucesivas victorias de la oposición en la década de 1990 y la alternancia en el Ejecutivo Federal en el año 2000, año en el que consiguió la victoria el Partido de Acción Nacional (PAN), cuando los analistas políticos comenzaron a interesarse realmente por la materia electoral en México. Además nuevos actores políticos han tomado posiciones en la arena política como son los empresarios; del mismo modo las clases medias y populares urbanas se han reorganizado y han tomado posturas más participativas.

La transformación social y política mexicana no puede ser explicada desde una sola perspectiva, sino que es necesaria una lectura multidisciplinar, aunque el hilo conductor sea el fenómeno electoral. Por ello, este libro se estructura en cuatro secciones introducidas por Víctor Alejandro Espinoza Valle y Luis Miguel Rionda Ramírez. El primer apartado se llama «México. Lecturas de la Transición Democrática»; en él se realiza 
un estudio comparando la teoría de las transiciones con la transición en México, determinándose una fecha clave, el 2 de julio de 2000, fecha que significa el fin de la transición democrática en México y el inicio de la nueva fase de instauración y consolidación de la democracia (César Cansino); se lleva a cabo un análisis de la economía política del país y de cómo interactúan la economía y la consolidación de la democracia (Rolando Cordera Campos); se analizan las fechas de inicio y fin de la transición, afirmando que son fechas difusas que como inicio se podría dar la fecha de la reforma de 1968, pero que no está tan claro que con la alternancia se haya puesto fin a la transición que todavía no se sabe que viene detrás de esa alternancia (Gilberto Rincón Gallardo); y se discute, de nuevo, si la transición ha terminado con la alternancia de Vicente Fox, se reconoce que ha sido un paso adelante pero que falta un verdadero proyecto que lleve a cabo una transformación de fondo en las instituciones políticas, judiciales y económicas del país, por lo que se determina que es una transición inacabada (Amalia García).

El segundo apartado se denomina «Partidos políticos y relación entre poderes». Se estudia el proceso de transformaciones que se han producido al interior de los partidos tras la alternancia del año 2000 para adaptarse a la nueva realidad política postautoritaria, modificándose elección interna, estatutos, etc. (Pablo Vargas González); plantea el alto grado de disciplina partidaria que se mantiene en México después de las elecciones de 2000, que es importante para determinar las relaciones entre el Ejecutivo y el Legislativo (Luis Carlos Ugalde) y se lleva a cabo un estudio de las relaciones del Ejecutivo y el Legislativo y la importancia de las mayorías en ambas Cámaras para su productividad comparando la legislatura actual y la anterior (Javier Hurtado).

El tercer apartado se denomina «Nueva competitividad electoral»; se afirma que en México la democratización está condicionada por las elecciones y el sistema de partidos al ser una democratización por medio de elecciones y para conseguirlo hay que deshegemonizar el sistema de partidos (Diego Reynoso); se examinan los cambios que se han producido en el sistema de partidos, que ha pasado a convertirse desde partido hegemónico en un pluralismo limitado con tres partidos principales (Juan Reyes del Campillo); se estudia la aparición de gobiernos divididos o gobiernos sin mayoría en el Congreso y de su impacto en la gobernabilidad democrática (Leonardo Valdés Zurita); se analiza si la existencia de municipios yuxtapuestos está relacionada con la concentración electoral en las regiones, con la competitividad y con la urbanización (Alain de Remes) y se analiza cómo pudo afectar la emergencia del Ejército Zapatista de Liberación Nacional en Chiapas al fortalecimiento de los partidos a nivel nacional (Silvia Gómez Tagle).

En el último apartado que se denomina «Cultura política y participación cívica», se plantea una propuesta teórico-metodológica para el estudio de la cultura política y de cómo los cambios producidos en ella han influido en el proceso democratizador en México (Héctor Tejera Gaona); se lleva a cabo un estudio sobre la relación entre los altos niveles de cultura cívica en Jalisco y el aumento del abstencionismo (Marco Antonio Cortés) y se analiza, a partir de la Encuesta Nacional de Juventud, la cultura cívica en 
los jóvenes de Baja California, territorio con un bajo empoderamiento de la ciudadanía (Alejandro Monsiváis Carrillo).

El trabajo es una compilación de las ponencias presentadas y reformadas tras su discusión en dos ciclos del Seminario Permanente sobre Procesos Electorales en México, el que se desarrolló a lo largo de 2002 que se denominó «Alternancia, Sociedad Civil y Democratización en México» y el llevado a cabo en 2003 que se denominó «Después de la Alternancia: Elecciones y Nueva Competitividad». Con este libro, se ha pretendido contribuir al mejor conocimiento de la dinámica del cambio político en México, por lo que se convierte en una obra de obligatoria consulta para los investigadores en la nueva realidad mexicana.

Amelia RODRÍGUEZ MarTíN 